\title{
BMJ Open Process evaluation of a randomised controlled trial of a pharmacological strategy to improve hypertension control: protocol for a qualitative study
}

\author{
Abdul Salam, ${ }^{1}$ Ruth Webster, ${ }^{2}$ Anushka Patel, ${ }^{2}$ Pavithra Godamunne, ${ }^{3}$ \\ Arunasalam Pathmeswaran, ${ }^{4} \mathrm{H}$ Asita de Silva, ${ }^{5}$ Anthony Rogers, ${ }^{2}$ Stephen Jan, ${ }^{2}$ \\ Tracey-Lea Laba ${ }^{6}$
}

To cite: Salam A, Webster $R$, Patel A, et al. Process evaluation of a randomised controlled trial of a pharmacological strategy to improve hypertension control: protocol for a qualitative study. BMJ Open 2018;8:e022317. doi:10.1136/ bmjopen-2018-022317

- Prepublication history for this paper is available online. To view these files, please visit the journal online (http://dx.doi org/10.1136/bmjopen-2018022317).

Received 14 February 2018 Revised 10 July 2018 Accepted 13 July 2018

Check for updates

(C) Author(s) (or their employer(s)) 2018. Re-use permitted under CC BY-NC. No commercial re-use. See rights and permissions. Published by BMJ.

For numbered affiliations see end of article.

Correspondence to

Dr Abdul Salam;

asalam@georgeinstitute.org.in

\section{ABSTRACT}

Introduction Globally, the prevalence of uncontrolled hypertension is high, particularly in low- and middleincome countries. There is a critical need for strategies to improve hypertension control. The early use of a fixed lowdose combination of three antihypertensive drugs (triple pill) has the potential to significantly improve hypertension control. The TRI ple Pill vs. $\mathbf{U}$ sual care $\mathbf{M}$ anagement for $\mathbf{P}$ atients with mild-to- moderate $\mathbf{H}$ ypertension (TRIUMPH) randomised controlled trial (RCT) is designed to test the effects of this strategy compared with usual care in patients with mild-to-moderate hypertension. This paper reports the protocol of a process evaluation of the TRIUMPH RCT. The objectives are to understand factors related to implementation of the intervention, mechanisms of effect, contextual factors that underpin the effectiveness of the triple pill strategy and the potential barriers and facilitators to implementing the strategy in clinical practice. Methods and analysis Face-to-face semistructured in-depth interviews with a purposive sample of TRIUMPH RCT participants and healthcare professionals in Sri Lanka will be conducted. Healthcare professionals will include physicians and their staff who were involved in conducting the TRIUMPH RCT. Interviewees will be recruited sequentially until thematic saturation is achieved. Interviews will be audio recorded, transcribed verbatim and analysed in NVivo using framework analysis methods. Ethics and dissemination The TRIUMPH RCT and process evaluation have received approval from the relevant Ethics Review Committee. All participants will be asked to provide written consent before participation. Findings from the study will be disseminated through publications and conference presentations.

Trial registration number ACTRN12612001120864, SLCTR/2015/020 ; Pre-results.

\section{INTRODUCTION}

High blood pressure (BP) is the number one preventable cause of early loss of life worldwide. ${ }^{1}$ Consequently, there has been much attention on efforts to prevent and treat high BP to avert premature deaths. Interventions that have been tested to improve
Strengths and limitations of this study

- Multiple sampling strategies to recruit a large number of interviewees to cover diverse views.

- Use of rigorous methods of data collection and analysis following a standard protocol.

- First of its kind study to explore barriers to hypertension control in Sri Lanka.

- Inability to recruit participants who did not attend the end of study visit.

hypertension control range from population-wide approaches such as national policies to reduce salt intake ${ }^{2}$ to radical and individual-based approaches such as renal denervation. ${ }^{3}$ By far, pharmacological treatment has been the most widely tested and is one of the most effective individual medical approaches to BP control.

A myriad of drugs with distinct and complementary physiological effects are at the disposal of physicians for prescription. Yet one in three people treated for hypertension have uncontrolled BP $(>140 / 90 \mathrm{~mm} \mathrm{Hg}) .{ }^{1}$ The current approach to the pharmacological treatment of hypertension commonly known as stepped care strategy involves initiation of monotherapy, with treatment intensification (dose uptitration or adding other drugs) at subsequent follow-up visits. While guidelines recognise that most patients need multiple drugs to achieve BP control, ${ }^{45}$ most patients receive only monotherapy. ${ }^{6}$

There are many patient- and provider-related factors relating to this stepped care strategy that might contribute to inadequate BP control. For instance, the cost and inconvenience of multiple clinic visits and treatment modification, 78 and non-adherence to treatment, which is worsened by increasing number of drugs ${ }^{9}$ are major determinants 
to BP control. In addition, therapeutic inertia, the resistance of prescribers to intensify therapy in the face of uncontrolled hypertension, is a recognised problem ${ }^{10} 11$ that is difficult to overcome. While fixed-dose combination (FDC) drugs are promising solutions to such issues, ${ }^{12}$ their use in clinical practice is primarily reserved for patients already established on multiple treatments, or more rarely, in the early stages of treatment for patients with markedly elevated $\mathrm{BP}^{45}$

The use of an FDC comprising three antihypertensive drugs in low doses (triple pill) as early or initial treatment has the potential to significantly improve hypertension control. The rationale is based on sound pharmacological principles: each drug will act on distinct and major physiological pathways of hypertension producing significant reduction in BP, and the use of low doses minimises adverse effects. Furthermore, its early use will achieve faster BP control and obviate the need for multiple clinic visits for treatment modification. However, the use of this strategy in clinical practice requires a shift in paradigm as it entails significant change in the prescribing behaviours of doctors, acceptance by the patients and regulatory approval. High quality evidence of its effectiveness and the reasons for its success or failure are needed.

The TRIUMPH trial is a parallel-group, open-label, randomised controlled trial (RCT) designed to test the effectiveness of early use of triple pill compared with usual care for the management of hypertension. ${ }^{13}{ }^{14}$ In brief, patients from multiple outpatient clinics in Sri Lanka, with mild-to-moderate hypertension, either naive to BP lowering drugs or receiving monotherapy were to be randomised to treatment with triple pill or continued usual care, for a period of 6 months. The primary outcome of the TRIUMPH RCT is improvement in the proportion of people with BP control. Secondary outcomes include reduction in $\mathrm{BP}$, improvement in adherence and improvement in quality of life. The TRIUMPH RCT has recruited 700 participants.

While an RCT assesses the effects of intervention on predefined outcomes, a process evaluation helps to understand aspects of delivery and receipt of intervention, why and how the intervention worked, influence of the settings in which the intervention was delivered and potential barriers and facilitators to implementing the intervention into clinical practice. ${ }^{15}$ Additionally, a process evaluation can help formulate hypotheses leading to further analyses of data from the RCT.

This paper presents the protocol for the process evaluation of a pragmatic RCT of a strategy of initial or early use triple pill compared with usual care for the management of hypertension among adults in Sri Lanka.

\section{METHODS AND ANALYSIS}

A logic model summarising the public health problem, evidence, resources, activities and anticipated outcomes of TRIUMPH study is depicted in figure 1. This logic model helps in understanding various components of the planned intervention, the resources in place, and sensitises us to potential interaction between these to produce the change. It also helps us to consider unintended influences on the trial results. ${ }^{15}$

\section{Study design and sample}

The process evaluation will employ qualitative methods of data collection and analysis and will involve semistructured in-depth interviews with trial participants and healthcare professionals. Interviewees will be recruited, initially, following a mixed purposive sampling strategy, including maximum variation, extreme or deviant case ${ }^{16}$ and subsequently by theoretical sampling to help explore themes that arise during initial analysis. ${ }^{17}$ Maximum variation sampling will consider trial participants age, gender, treatment group, history of diabetes and cardiovascular diseases (CVD), income and lifestyle. Healthcare professionals will include physicians and their staff who participated in the TRIUMPH study. Interviews will be recruited sequentially until thematic saturation is achieved. From our previous experience, ${ }^{18} 19$ we anticipate achieving thematic saturation with 20-25 trial participant interviews (approximately 10-12 each treatment group) and 10-15 health professional interviews.

The study team will include researchers with diverse background: AS (pharmacist, quantitative and qualitative researcher), TL (pharmacist, health economics and qualitative researcher), RW (physician, quantitative researcher), PG (social sciences researcher), SJ (health economist, quantitative and qualitative researcher) and AP (cardiologist, quantitative researcher), with some being part of the core team of TRIUMPH RCT and others completely independent.

\section{Data collection}

Data collection will be done by means of semistructured in-depth interviews with individual participants and healthcare providers. This method has primacy over other qualitative methods in the context of this study as they allow in-depth inquiry of the phenomenon, ensures confidentiality of the participants and enables prespecified topics to be explored while permitting the exploration of other ideas and thoughts that may spontaneously arise in the conversation. ${ }^{20}$

Interviews with trial participants will begin after the first participant enrolled in TRIUMPH RCT has completed their 6-month follow-up (end of study) visit. Healthcare professional interviews will be conducted following the end of follow-up of all trial participants in the TRIUMPH RCT. Consenting trial participant and healthcare professionals will be invited to an interview at the trial site or other place of their choice. Staff at TRIUMPH RCT sites will be notified of the identity of the trial participants nearer her/his end of study site visit. On the day of end of study visit, selected participants will be asked to confirm their willingness to be interviewed. The interviewer will discuss the objectives of the interview, and inform the interviewee of the potential 


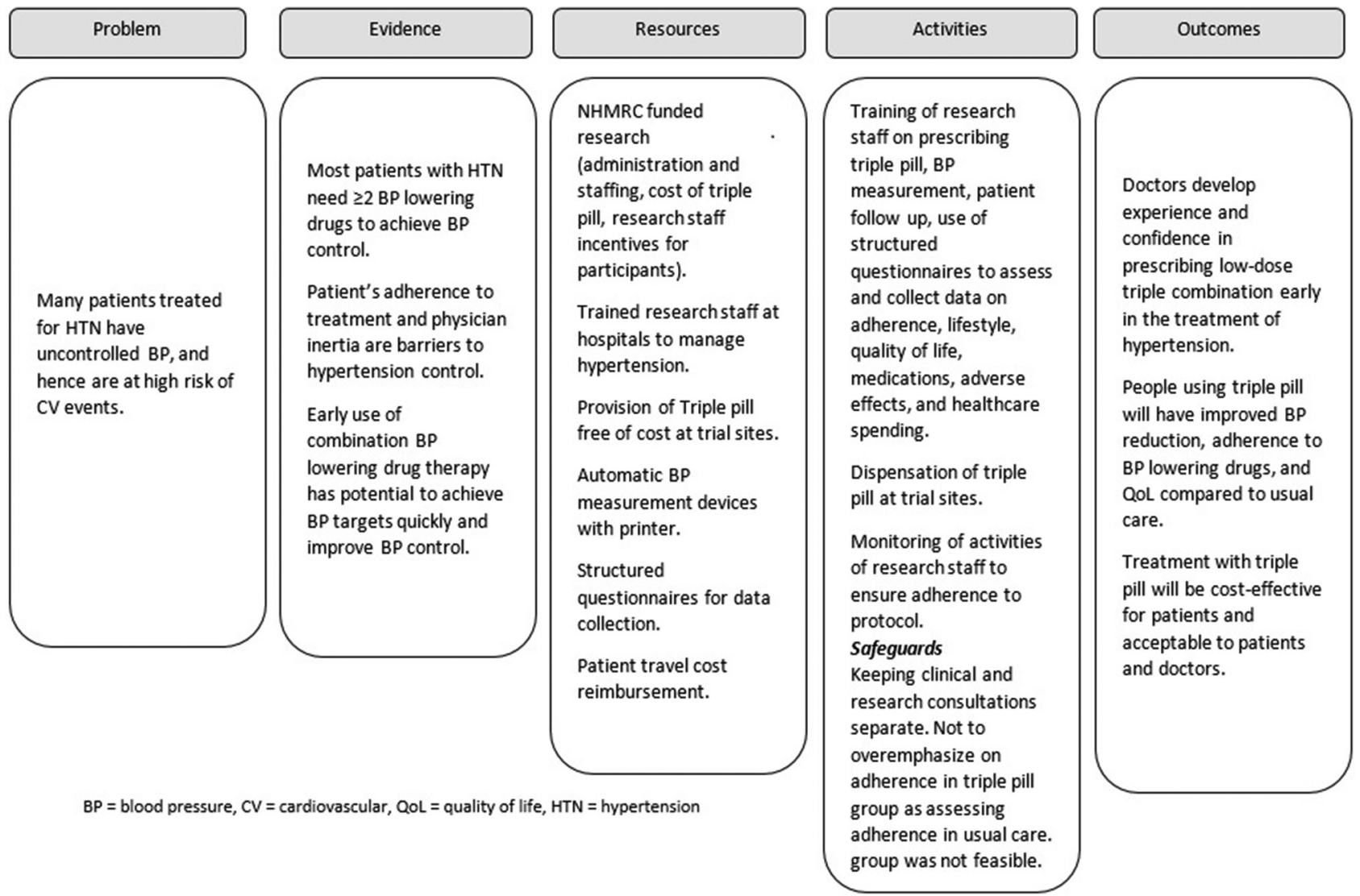

Figure 1 A logic model summarising the public health problem, evidence, resources, activities and anticipated outcomes of TRIUMPH study.

benefits and harm of participation, and provide assurances of confidentially. If willing to be interviewed, the study coordinator will organise a private place for the conduct of interview. Interviews will be conducted by trained interviewers in appropriate local language with trial participants and in English with healthcare professionals. Interviewees will be informed that there are no right or wrong answers and they are free to express their views in whichever manner they may want to. The interviewer will follow an interview guide, structuring questions based on the responses of the interviewee and ensuring that all the key areas mentioned in the interview guide are covered. Each interview is likely to last, on average, $30 \mathrm{~min}$ and will be audio recorded.

\section{Interview guides}

The research team will develop interview guides specific for trial participant and healthcare professional interviews. Guide development will be informed by the objectives of the process evaluation, a literature review of the topic under research and brain storming among the research team. Key areas that will be covered in the interview guides include views and experience of, the TRIUMPH RCT, triple pill and BP control, adherence to treatment, hypertension and its management in Sri Lanka and translation of the intervention into practice and policy. Interview guides will be piloted before being used for data collection. During the process evaluation, based on the contemporaneous analysis of collected data, interview guides will be subjected to amendments as necessary in discussion with the research team. ${ }^{21}$

\section{Data management and analysis}

Translation of interviews

All interviews will be transcribed verbatim. Non-English language interviews will then be translated to English by native translators. Each transcript will be then reviewed by the interviewers against the audio for accuracy of the transcription and translation. Any content which may reveal the identity of the interviewees will be removed from the transcript.

\section{Analysis}

Framework analysis, a qualitative data analysis method, is increasingly used across multiple disciplines: psychology, social and health science. It is particularly useful in addressing four types of research questions: contextual, diagnostic, evaluative and strategic. ${ }^{22}$ The research questions of our study fall in more than one of these categories. Further, the other aspects of our research, clearly set objectives, short time scale, makes framework analysis a preferred approach to data analysis. 
Each transcript will be read thoroughly by two researchers, for data familiarisation, before coding the text. Using Nvivo, ${ }^{23}$ two researchers will independently code the first few, same, interview transcripts. The team of investigators include experts from Sri Lanka to help understand the nuances of local language wherever required. This initial coding will involve careful review of text, line-by-line, to generate as many relevant codes as possible from different perspectives without much regard to the objectives of the study. The two coders will then compare and discuss codes to develop a framework of codes and their categories that will be applied to subsequent transcripts, without any restriction on generating new codes. The coding of the rest of the transcripts will proceed following the coding framework. The coding framework will be subjected to modification if necessary, by discussion among the coders, as the coding of interview transcripts proceed. Throughout the process of data management and analysis, researchers will draft memos of reflections, ideas and interpretations, and discuss them with the team to inform analysis.

Using Microsoft Excel, a framework matrix will be created with the names of the categories of codes as column headings and interviewee identification number on the rows. Each category of nodes will be summarised ensuring its essence is intact, and mapped in the matrix. From the framework matrix, we will compare themes across interviewees, identify patterns and connection between the categories, identify divergent themes and generate memos of the rich description of the phenomena relevant to the objectives of the study.

\section{PATIENT AND PUBLIC INVOLVEMENT}

Patients were not involved in the design of the study. Although we did not have a patient partner formally involved, the research question and outcome measures were substantially informed by our former research about patient experience and preferences for combination CVD therapies. Results of the study will be disseminated to study participants through healthcare professionals in our research network.

\section{ETHICS AND DISSEMINATION Dissemination}

The results of this study, including the framework matrix and themes arising from the interviews, will be shared with the individuals and institutions associated with this study as well as academic audiences through peerreviewed publication and conference presentations.

This process evaluation will complement and add value to the TRIUMPH trial by providing a better understanding of trial results. It will provide insights into the relevance, usefulness and adaptability of the strategy into clinical practice in Sri Lanka as well as other low- and middle-income settings. Improvement in BP control is dependent on several factors related to patient, provider, therapy and healthcare system. Therefore, understanding of implementation of the strategy and its interaction with these factors will also help understand determinants of BP control.

\section{Study status}

Data collection commenced in May 2017 and is likely to end in May 2018.

\section{Author affiliations}

${ }^{1}$ The George Institute for Global Health, University of New South Wales, Hyderabad, India

${ }^{2}$ The George Institute for Global Health, University of New South Wales, Sydney, Australia

${ }^{3}$ Department of Medical Education, Faculty of Medicine, University of Kelaniya, Kelaniya, Sri Lanka

${ }^{4}$ Department of Public Health, Faculty of Medicine, University of Kelaniya, Kelaniya, Sri Lanka

${ }^{5}$ Clinical Trials Unit, Department of Pharmacology, Faculty of Medicine, University of Kelaniya, Kelaniya, Sri Lanka

${ }^{6}$ Menzies Centre for Health Policy, Sydney Medical School, The University of Sydney, Camperdown, New South Wales, Australia

Contributors AS, T-LL, RW, AP, AR, SJ: conceived and designed the study. PG, APS, HAdeS: contributed to the design of the study and are responsible for study coordination. AS, T-LL: drafted the manuscript. All authors reviewed the article and provided approval for the final version of the manuscript submitted to the journal for publication.

Funding This study is funded by the National Health and Medical Research Council (NHMRC) Australia, (App: 1041052) through The Global Alliance for Chronic Diseases (GACD) Hypertension prevention and control, funding scheme.

Competing interests George Health Enterprises, the social enterprise arm of The George Institute for Global Health, has received investment to develop fixed-dose combination products containing aspirin, statin and blood pressure lowering drugs. George Health Enterprises has submitted patents for low-dose blood pressure combinations, on which AR is listed as one of the inventors.

Patient consent Not required.

Ethics approval Ethics Review Committee, Faculty of Medicine, University of Kelaniya, Sri Lanka, and Ethics Review Committee (RPAH Zone), Sydney, Australia.

Provenance and peer review Not commissioned; externally peer reviewed.

Open access This is an open access article distributed in accordance with the Creative Commons Attribution Non Commercial (CC BY-NC 4.0) license, which permits others to distribute, remix, adapt, build upon this work non-commercially, and license their derivative works on different terms, provided the original work is properly cited, appropriate credit is given, any changes made indicated, and the use is non-commercial. See: http://creativecommons.org/licenses/by-nc/4.0/.

\section{REFERENCES}

1. Chow CK, et al. Prevalence, awareness, treatment, and control of hypertension in rural and urban communities in high-, middle-, and low-income countries. JAMA 2013;310:959-68.

2. Webster JL, Dunford EK, Hawkes C, et al. Salt reduction initiatives around the world. J Hypertens 2011;29:1043-50.

3. Bhatt DL, Bakris GL. Renal denervation for resistant hypertension. N Engl J Med 2014:371:184.

4. Whelton PK, Carey RM, Aronow WS, et al. 2017 ACC/AHA/AAPA/ ABC/ACPM/AGS/APhA/ASH/ASPC/NMA/PCNA guideline for the prevention, detection, evaluation, and management of high blood pressure in adults: a report of the American College of Cardiology/ American Heart Association Task Force on Clinical Practice Guidelines. J Am Coll Cardiol 2017;24430.

5. Mancia G, Fagard R, Narkiewicz K, et al. 2013 ESH/ESC guidelines for the management of arterial hypertension: the Task Force for the Management of Arterial Hypertension of the European Society 
of Hypertension (ESH) and of the European Society of Cardiology (ESC). Eur Heart J 2013;34:2159-219.

6. Chow CK, Teo KK, Rangarajan S, et al. Prevalence, awareness, treatment, and control of hypertension in rural and urban communities in high-, middle-, and low-income countries. JAMA 2013;310:959-68.

7. Hughes D, McGuire A. The direct costs to the NHS of discontinuing and switching prescriptions for hypertension. $J$ Hum Hypertens 1998;12:533-7.

8. McCombs JS, Nichol MB, Newman CM, et al. The costs of interrupting antihypertensive drug therapy in a Medicaid population. Med Care 1994;32:214-26.

9. Gupta P, Patel P, Štrauch B, et al. Risk factors for nonadherence to antihypertensive treatment. Hypertension 2017;69:1113-20.

10. Kjeldsen SE, Julius S, Dahlöf B, et al. Physician (investigator) inertia in apparent treatment-resistant hypertension - insights from large randomized clinical trials. Lennart Hansson Memorial Lecture. Blood Press 2015;24:1-6.

11. Harle CA, Harman JS, Yang S. Physician and patient characteristics associated with clinical inertia in blood pressure control. J Clin Hypertens 2013;15:820-4.

12. Gupta AK, Arshad S, Poulter NR. Compliance, safety, and effectiveness of fixed-dose combinations of antihypertensive agents: a meta-analysis. Hypertension 2010;55:399-407.

13. Salam A, Webster R, Singh $K$, et al. TRIple pill vs usual care management for patients with mild-to-moderate hypertension (TRIUMPH): Study protocol. Am Heart J 2014;167:127-32.
14. Webster R. Protocol changes to the TRIUMPH study. Am Heart $J$ 2017;191:e1.

15. Moore GF, Audrey S, Barker M, et al. Process evaluation of complex interventions: Medical Research Council guidance. BMJ 2015;350:h1258.

16. Patton MQ. Qualitative evaluation and research methods: SAGE Publications, inc, 1990.

17. Bazeley P. Qualitative data analysis: practical strategies: Sage, 2013.

18. Salam A, Stewart F, Singh K, et al. INterpreting the Processes of the UMPIRE Trial (INPUT): protocol for a qualitative process evaluation study of a fixed-dose combination (FDC) strategy to improve adherence to cardiovascular medications. BMJ Open 2013;3:e002313.

19. Wood F, Salam A, Singh K, et al. Process evaluation of the impact and acceptability of a polypill for prevention of cardiovascular disease. BMJ Open 2015;5:e008018.

20. Brinkmann S. Qualitative interviewing: Oxford university press, 2013.

21. Given LM. 100 questions (and answers) about qualitative research: SAGE Publications, 2015

22. Ritchie J, Spencer L. Qualitative data analysis for applied policy research. In: Michael Huberman A, Miles MB, The qualitative researcher's companion: SAGE, 2002;573:305-29.

23. QSR International Pty Ltd. NVivo qualitative data analysis software; version 10. Melbourne: QSR International, 2012. 